Revue des patrimoines

$21 \mid 2013$

De l'art de bâtir aux champs à la ferme moderne

\title{
François Cointeraux (1740-1830). L'avant-garde de Art de bâtir aux champs
}

Jean-Philippe Garric

\section{OpenEdition}

Journals

Édition électronique

URL : http://journals.openedition.org/insitu/10345

DOI : $10.4000 /$ insitu. 10345

ISSN : 1630-7305

Éditeur

Ministère de la culture

Référence électronique

Jean-Philippe Garric, « François Cointeraux (1740-1830). L'avant-garde de Art de bâtir aux champs », In Situ [En ligne], 21 | 2013, mis en ligne le 12 juillet 2013, consulté le 02 mai 2019. URL : http:// journals.openedition.org/insitu/10345; DOI : 10.4000/insitu.10345

Ce document a été généré automatiquement le 2 mai 2019.

\section{(c) $($ i) $(9)$}

In Situ Revues des patrimoines est mis à disposition selon les termes de la licence Creative Commons Attribution - Pas d'Utilisation Commerciale - Pas de Modification 4.0 International. 


\title{
François Cointeraux (1740-1830). L'avant-garde de Art de bâtir aux champs
}

\author{
Jean-Philippe Garric
}

1 Dans une précédente intervention consacrée aux mutations qui marquent les débuts de la période contemporaine dans le domaine du livre d'architecture ${ }^{1}$, j'avais proposé François Cointeraux comme l'un des exemples pouvant illustrer l'émergence d'une relation nouvelle entre l'architecture et l'imprimé, autour de 1800. Je faisais ainsi l'hypothèse selon laquelle les publications d'architecture devaient être envisagées comme une dimension à part entière de l'architecture et un terrain aussi propice que les projets et les réalisations bâties pour étudier cette discipline. Je voudrais ici développer ce point de vue, dans une perspective méthodologique qui est celle de la bibliographie matérielle: une approche qui accorde une importance égale aux contenus et à la forme des ouvrages, en développant une analyse du livre d'architecture, qui s'intéresse autant à l'histoire du livre qu'à celle de l'architecture.

2 Thomas de Quincey, dans De l'assassinat considéré comme un des beaux-arts, évoque le voyage en Angleterre du Lyonnais Louis Simond, qui non seulement était un compatriote de François Cointeraux mais qui était aussi à quelques années près son contemporain. «Voici une quarantaine d'années, écrit le Britannique opiomane, un Français, M. Simond, dans ses « Voyages », mentionne incidemment l'horrible scène suivante comme ayant été dûment observée de ses yeux dans la chevaleresque France, peu de temps avant la Révolution Française. Un paysan était en train de labourer, et l'attelage qui tirait sa charrue se composait d'un âne et d'une femme. Cela est suffisamment attristant ; mais le Français ajoute qu'en distribuant ses coups de fouet, le paysan était évidemment désireux de se montrer impartial ; ou que, si l'un des deux compagnons du joug avait à se plaindre, ce n'était certainement pas l'âne $»^{2}$.

3 De Quincey enjolive un peu la citation. Le Français, qui venait de dénoncer la brutalité avec laquelle on pouvait traiter l'autre moitié de l'humanité dans certaine partie reculée de l'Écosse, entendait toutefois relativiser sa critique en reconnaissant que son propre 
pays n'était pas exempt de critique, sur le même terrain. Le passage exact est le suivant: «Au reste, j'ai vu plus d'une fois dans les guérets de la Gaule amoureuse une femme et un âne attelés à la même charrue et le laboureur en guenilles hâtant son attelage d'un fouet impartial. Ainsi, concluait-il, il ne faut pas nous moquer des étrangers $»^{3}$.

On hésite à décider si cette scène relève d'une dénonciation indignée de la barbarie régnant alors dans les campagnes - on pense à la notion de tanière évoquée par JeanMarie Pérouse de Montclos ${ }^{4}$ ou plutôt de la farce rustique, ou encore d'un sous-entendu érotique sadien, ou des trois à la fois. Plus généralement, elle renvoie à la relation à la fois ethnographique, philanthropique et intéressée que les élites de la fin du XVIII et du début du XIX ${ }^{e}$ siècle entretenaient avec un monde agricole qu'elles aspiraient à réformer pour assurer son exploitation dans des conditions plus harmonieuses. Évoquant, à l'inverse, cette déclaration humaniste du comte de Lasteyrie, selon laquelle «plus un peuple est libre, mieux il traite les animaux ».

5 Ce n'est pas ce point de vue surplombant, à la fois critique, paternaliste et distancié qu'adopte François Cointeraux, lorsqu'il se propose de contribuer au progrès du monde agricole. L'ancien maçon lyonnais revendique, au contraire, une démarche fondée sur sa propre expérience. Il se présente non pas comme un observateur et un réformateur qui aborderait de l'extérieur les problèmes dont il s'empare, mais bien comme l'un de ceux dont il prétend améliorer la vie. Fort de sa double familiarité avec le monde de l'agriculture et avec celui du bâtiment, c'est en spécialiste de l'Art de bâtir aux champs qu'il s'investit - à travers ses publications - d'une mission au service de la communauté tout entière : un grand but dont la réussite, à ses yeux, dépendrait uniquement de la confiance que pourraient lui accorder les pouvoirs publics et de leur capacité à le seconder de façon satisfaisante :

6 "Il manque donc au public, écrivait-il ainsi en 1793, un homme qui ait fait remuer à la fois la truelle \& le marteau, avec la bêche et la houe? Je me présente pour remplir ces deux fonctions, \& je ferai voir que l'on peut, en améliorant un fond, construire une petite bâtisse : je tirerai parti de la science même du vigneron, du laboureur, du jardinier, pour en faire des bâtisseurs.

7 C'en est assez : si l'on trouve mon dévouement au bien de la chose publique, \& mes talents particuliers utiles? je demande alors le concours unanime pour acheter mes petits ouvrages, \& je dois l'attendre des départemens, districts \& communautés : ce seront ces corps qui peuvent soutenir mon entreprise \& ne pas la laisser échouer : Ce ne sera pas ma faute si cela arrive, car, pour moi je ferai tout mon possible pour servir mes compatriotes, $\&$ porter à la perfection le traité d'architecture rurale $»^{5}$.

8 Au titre des qualités dont il se pare ainsi, François Cointeraux passe cependant sous silence son penchant littéraire et son talent de publiciste, son sens de la formule et son activisme éditorial, qui ne sont pourtant, sans doute, ni les moindres facteurs de sa réussite, ni la moindre motivation de son engagement. Avec le progrès des techniques d'exploitation, les mutations de la propriété foncière consécutives à la vente des biens nationaux, et l'émergence d'une réflexion rationnelle sur les constructions agricoles, la Révolution française contribuait fortement à une véritable démocratisation de l'architecture dans les villes comme dans les campagnes. Les urbains qui, de plus en plus nombreux, investissaient dans l'agriculture des fortunes issues du commerce ou de l'industrie, devenaient ainsi les propriétaires d'un patrimoine bâti et les responsables des populations qui allaient avec, acquérant en même temps qu'une certaine notabilité locale, 
la possibilité de prendre en main la gestion ou la construction d'édifices. Les propriétaires plus anciens se trouvaient pris dans ce mouvement de réforme et de modernisation sociale et économique du monde agricole et de ses constructions.

9 Mais, pour exercer cette maîtrise d'ouvrage, ils ne disposaient pas toujours de véritables architectes et ils devaient donc se tourner vers des maçons, des entrepreneurs ou des ingénieurs, qui n'avaient pas toujours acquis une culture savante très approfondie. Mais ils pouvaient aussi s'appuyer, à mesure que l'on avançait dans le XIX ${ }^{\mathrm{e}}$ siècle, sur des livres d'architecture spécialisés de plus en plus nombreux. La diffusion d'une culture architecturale par l'imprimé accompagnait l'accès d'un plus grand nombre à l'architecture.

10 Inversement, l'autre face de ce mouvement de démocratisation de l'imprimé d'architecture est que davantage de personnes pouvaient désormais accéder au statut d'auteur dans le champ de l'architecture et le revendiquer. Les prérogatives de l'écriture, assez étroitement liées du reste à celles de l'enseignement et qui étaient jadis réservées à quelques académiciens, quelques lettrés, ou quelques architectes d'exception, pouvaient désormais être partagées par des hommes de plus en plus nombreux, y compris parmi ceux que leurs origines ne prédisposaient pas naturellement à l'écriture.

11 Cointeraux, qui provenait lui-même d'un milieu qui n'était pas par tradition celui des lettres et des livres occupe une place à part, et désormais largement reconnue, parmi ces auteurs nouveaux qui investissent ainsi le champ de la théorie de l'architecture pour tous. Son sujet de prédilection et son grand combat étaient la construction en terre crue et tout ce qui pouvait s'y rapporter. La plus grande partie de ses livres et de ses opuscules, qui sont près d'une centaine, porte sur la construction des murs en pisé - c'est-à-dire en terre battue entre deux banches - mais également sur la préfabrication de briques, une de ses inventions qualifiée de "nouveau pisé », et accessoirement sur la transformation des pressoirs à vin et pressoirs à terre, sur la peinture permettant de recouvrir et d'orner les constructions ainsi construites, sur les murs de clôture employant ce matériau, ainsi que les murs de terrasse. Cet engagement est l'aspect de sa production qui a suscité le plus d'intérêt. Il a été considéré à la fois comme un précurseur du béton et comme le premier défenseur d'une construction alternative, employant un matériau gratuit et inépuisable, depuis les études de la fin des années 1970 liées à l'intérêt contemporain pour les architectures de terre, jusqu'aux travaux les plus récents conduits dans le domaine de l'histoire des techniques, jusqu'à l'article récent de Laurent Baridon ${ }^{6}$, qui s'attache à replacer cette contribution au progrès de l'art de bâtir dans une perspective d'histoire sociale (fig. $\left.\mathbf{n}^{\circ} \mathbf{1}\right)$. 

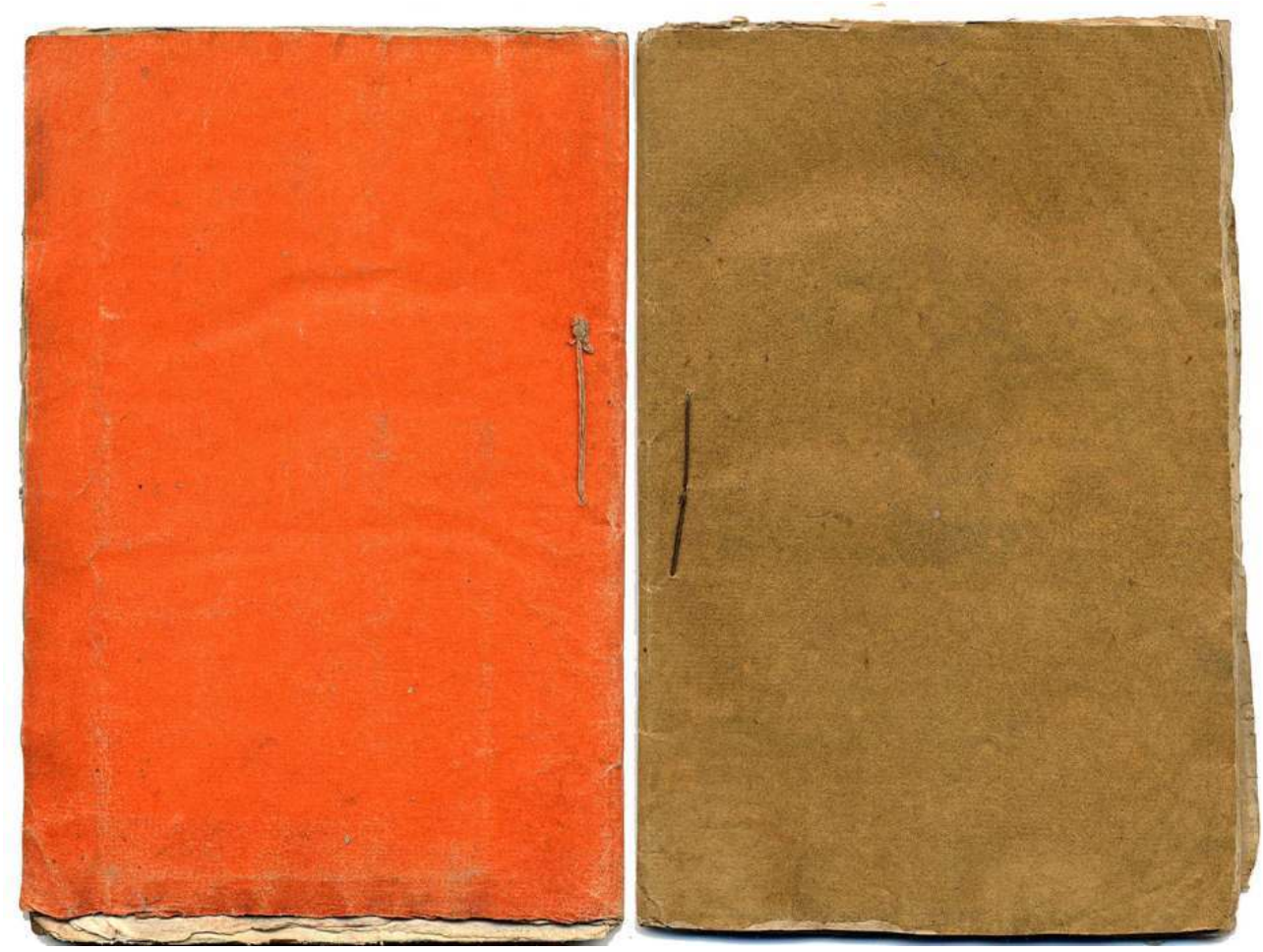

DEUX DES NOMBREUX FASCICULES ÉCRITS, ÉDITÉS ET VENDUS PAR FRANÇOIS COINTERAUX, ENCORE REVÊTUS DE LEURS COUVERTURES D'ORIgINE.

REPRO. GARRIC, JEAN-PHILIPPE. (C) JEAN-PHILIPPE GARRIC.

Les recherches de Cointeraux sur l'économie du bois de chauffage, qui est son autre thématique récurrente, ont également retenu l'attention des spécialistes et les travaux récents de Louis Cellauro et Gilbert Richaud ont permis de démontrer notamment que son audience internationale avait été considérable ${ }^{7}$. Mais ses préoccupations s'étendaient à de nombreux autres sujets et même à de nombreux domaines, au-delà des seules constructions agricoles et des architectures rurales. Certains concernent directement l'agriculture, comme les clôtures fertilisantes (cat. 54.A), ou la façon de fumer les terres en employant une autre de ses nouvelles inventions servant tout à la fois de charrue, de herse et de rouleau (cat. 54.B), ou encore la manière de tirer du sucre du jus de raisin, qu'il expose dans Le joyeux vigneron à ses vendanges (cat. 57). On peut encore évoquer les études qu'il consacre successivement à différents programmes pour les améliorer : fermes (cat. 3), bergeries (cat. 26), faisanderies (cat. 27), basses-cours (cat. 54.G), mais aussi manufactures (cat. 2.C) et maisons de campagne (cat. 2.C). D'autres concernent la vie des paysans et l'aménagement de l'intérieur de leurs habitations. C'est le cas de La cuisine renversée ou le nouveau ménage (cat. 16) un ouvrage de soixante-douze pages qu'il publie, en 1796, exceptionnellement non pas sous son seul nom mais sous la signature collective de «La famille Cointeraux », ou de la conférence de 1811 intitulée Le journalier à son aise ou l'art d'incorporer les meubles dans l'immeuble (cat. 54.J), où il défend l'idée de la disparition du mobilier (fig. $\mathbf{n}^{\circ} \mathbf{2}$ ). 
Figure 2

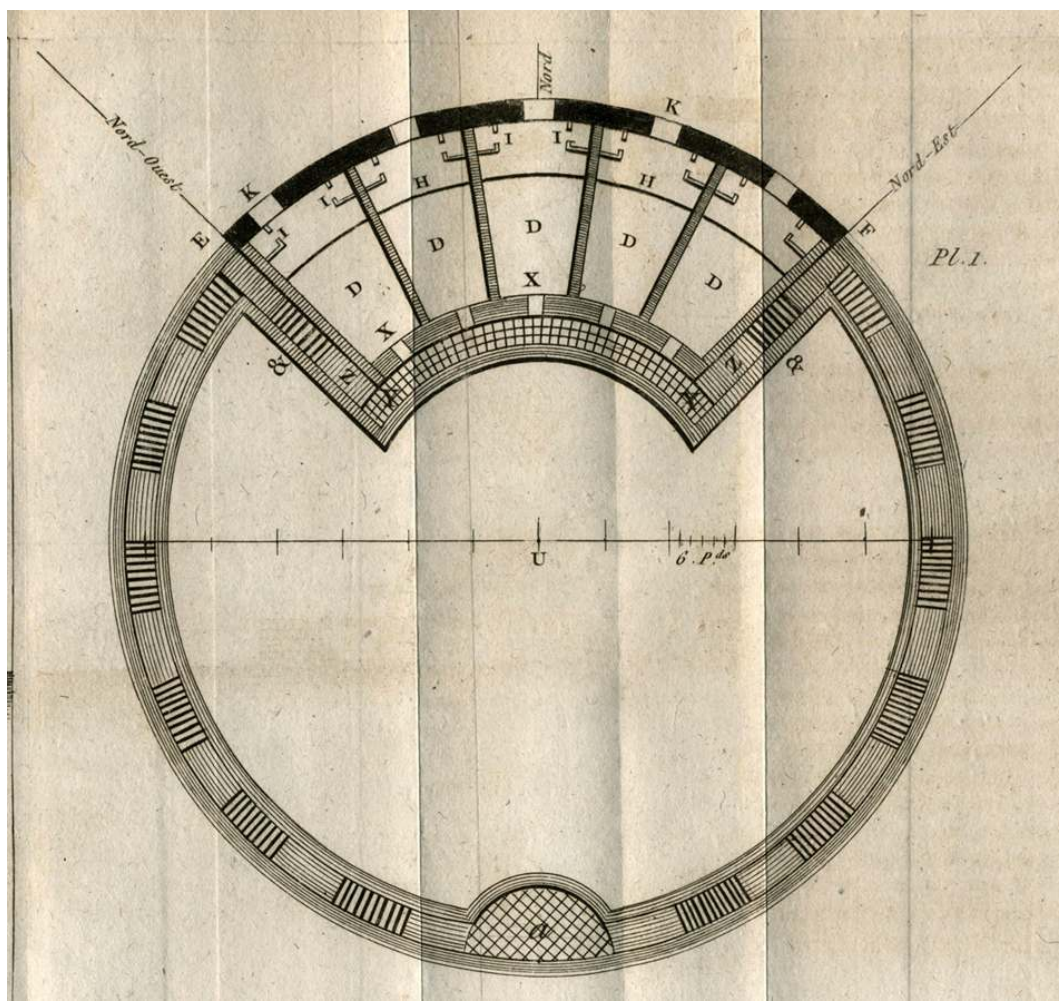

Plan d'une faisanderie, Nouvelles dispositions de faisanderies, 1805.

REPRO. GARRIC, JEAN-PHILIPPE. @ JEAN-PHILIPPE GARRIC.

Cependant Cointeraux ne limite pas ses ambitions à des questions pratiques touchant l'architecture rurale et le monde agricole. Dans un genre plus relevé, il fait imprimer en 1798 et 1799 plusieurs ouvrages sur Paris, l'un à caractère à la fois historique et prospectif : Paris tel qu'il était à son origine; Paris tel qu'il est aujourd'hui... avec un projet de l'auteur sur divers embellissements dont cette ville est susceptible (cat. 9), les deux autres relevant plutôt de la critique architecturale : L'art d'embellir le Louvre et les Tuileries (cat. 18) et Réflexions impartiales sur l'odéon (cat.20) qui était distribué gratis à tous les acheteurs des autres livres de l'auteur sur Paris.

Certaines de ses réflexions sont même plus franchement théoriques, comme celles qui concernent la pente des toits, «à l'usage des différents peuples » (cat. 23), ou encore la "théorie de la surface du globe terrestre appliquée aux besoins de l'homme » (cat. 54.C) (fig. $\mathbf{n}^{\circ} 3$ ). 


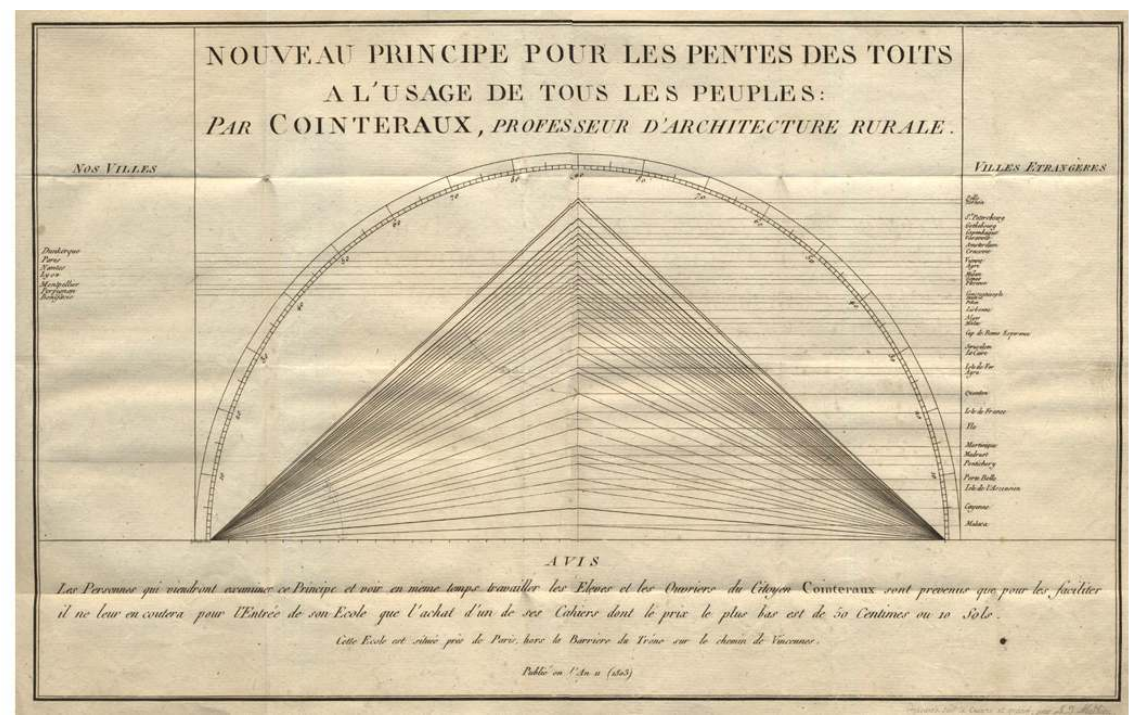

Nouveau principe pour les pentes des toits à l'usage de tous les peuples

REPRO. GARRIC, JEAN-PHILIPPE. @ JEAN-PHILIPPE GARRIC.

Il faut y ajouter des publications plus opportunistes, comme celles qui sont consécutives à la mise en place des nouvelles mesures et du nouveau calendrier : l'Almanach perpétuel et républicain des cultivateurs ou la Méthode familière pour apprendre en peu de temps les nouveaux poids et les nouvelles mesures républicaines (cat. 13), qui semblent démontrer que ses choix éditoriaux n'étaient pas toujours dénués de visées commerciales. Laurent Baridon estime que cette activité éditoriale pouvait lui rapporter quelques subsides, mais cette dimension économique de l'activité de Cointeraux est incertaine, encore méconnue et plutôt insaisissable. Entre les revenus qu'il pouvait tirer de biens acquis précédemment, la commercialisation de ses ouvrages, les droits d'entrée à ses conférences, les travaux qu'il se voyait confier et les subventions qu'il réclamait activement et obtenait parfois on ignore de quoi se composaient les revenus de François Cointeraux. Tout juste peut-on observer que sa principale commande officielle connue: une participation à la construction de la Roche-sur-Yon à partir du mois d'avril 1806, correspond à une accélération importante de son activité éditoriale, puisqu'il publie pas moins de dix opuscules cette année-là, plus une réédition de l'Avis au peuple (cat. 33), suivis de cinq nouveaux opus et douze " conférences » l'année suivante, ce qui semblerait indiquer que les éditions étaient davantage une dépense qu'une source de recettes.

Toutefois, à défaut de tenter d'approfondir cette question mal documentée, arrêtons-nous plutôt ici, non pas sur l'étude des idées et des inventions proposées par Cointeraux, mais sur ce qui fut le cœur même de son activité: son métier d'auteur, de publiciste et d'éditeur.

\section{Un professionnel de l'imprimé}

17 Ce que nous savons de lui nous le présente en effet comme un véritable professionnel de l'imprimé et cette dimension, qui contribue très largement à la fascination exercée par le personnage, ne peut certes pas être considérée comme un à-côté, un travers à la fois sympathique et pittoresque. Bien sûr son audace à s'emparer des thématiques les plus 
diverses peut prêter à sourire. L'homme, en effet, n'est pas un érudit et sa culture n'est certainement pas aussi encyclopédique qu'il le faudrait pour aborder une palette de sujets d'intérêt aussi variés qu'il ne le fait. Mais sa naïveté est largement compensée par son sens de la formule et par l'énergie qu'il déploie dans une activité constante d'invention, d'exposition, de conférence et de publication. Si bien que ce solitaire obstiné apparaitt rétrospectivement comme une avant-garde à lui seul.

Celui qui s'autoproclamait, parlant de lui à la troisième personne, «le professeur d'architecture rurale » et qui décida vers la cinquantaine, après une carrière de maçon lyonnais, d'entamer un parcours incertain de théoricien parisien, mena avec panache un combat enthousiaste, en multipliant sur un rythme effréné des publications conçues comme autant de manifestes, qui témoignent d'un sens de l'humour, du détournement et de la provocation, et qui renvoient à une activité permanente de démonstration, d'exposition et de conférences publiques.

\section{Publicité et pratiques commerciales}

19 La dimension la plus immédiatement frappante de l'œuvre de Cointeraux est sans doute l'effort qu'il accorde à en faire la publicité et son dynamisme en matière de pratiques commerciales. Les annonces et les articles concernant son activité inondent véritablement les périodiques contemporains, comme le Journal encyclopédique, La feuille de correspondance du libraire, Le journal général de la littérature de France, Le télégraphe littéraire, Les Annales des manufactures et des arts, ou le Journal des connaissances usuelles, autant de publications qui ne s'adressaient certainement pas en premier lieu aux agriculteurs, mais aussi des publications locales comme le Journal du département de la Vienne. Dans Le moniteur de la Convention du premier Thermidor an I, il présente son action comme républicaine et relevant de l'intérêt public : « Le professeur d'architecture rurale prévient le public, écrit-il, qu'il vient de faire graver plusieurs planches ; mais il aurait besoin d'un plus grand nombre de souscripteurs pour continuer les frais dispendieux de ces gravures. Les républicains qui voudront souscrire à ce journal d'agriculture et des arts auront aujourd'hui l'avantage de recevoir, beaucoup à la fois, des textes et des gravures. Le prix de ce journal est de 30 liv., qu'il faut adresser, franc de port, au citoyen Cointeraux, professeur d'architecture rurale, rue du Faubourg-Honoré, n 108 , en face de la Grande Rue Verte ». L'annonce extrêmement imprécise revenait pour ainsi dire à demander un blanc seing aux souscripteurs. Elle correspond apparemment à l'ouvrage intitulé Agriculture et architecture rurale (cat. 8 et 49) dans le prospectus duquel il était précisé : «Le Professeur d'Architecture Rurale, ayant un Traité complet à faire qui manque à toutes les Nations, tant sur l'Agriculture que sur l'Économie des Bâtimens, invite tous les bons Républicains à lui aider ». La seconde livraison s'intitulait «Du pot au feu des Sans-Culottes, des nouveaux logemens, des modes de l'occupation, etc. ", la troisième Almanach perpétuel et Républicain des Cultivateurs (cat. 8), quant à la quatrième, annoncée sous presse, elle concernait la culture des pommes de terre et les différents apprêts que l'on peut en faire dans la cuisine et pour les basses-cours. Dans un mouvement circulaire d'autopromotion permanente certains de ces articles et de ces annonces parus dans la presse étaient repris dans les fascicules, comme une preuve de son audience.

La publicité de ses éditions était également assurée par des catalogues et des prospectus imprimés, qu'on trouve le plus souvent reliés dans ses opuscules et qui annoncent des pratiques commerciales comme celle que développèrent les éditions Roret à partir des 
années 1820, et par des annonces commerciales ou promotionnelles omniprésentes dans ses publications, qu'elles s'adressent aux pouvoirs publics, ou aux organes de presse. Sur les couvertures imprimées de ses conférences de 1809, on peut suivre ses échanges commerciaux avec ses souscripteurs (fig. $\left.\mathbf{n}^{\circ} \mathbf{4}\right)$.

Figure 4

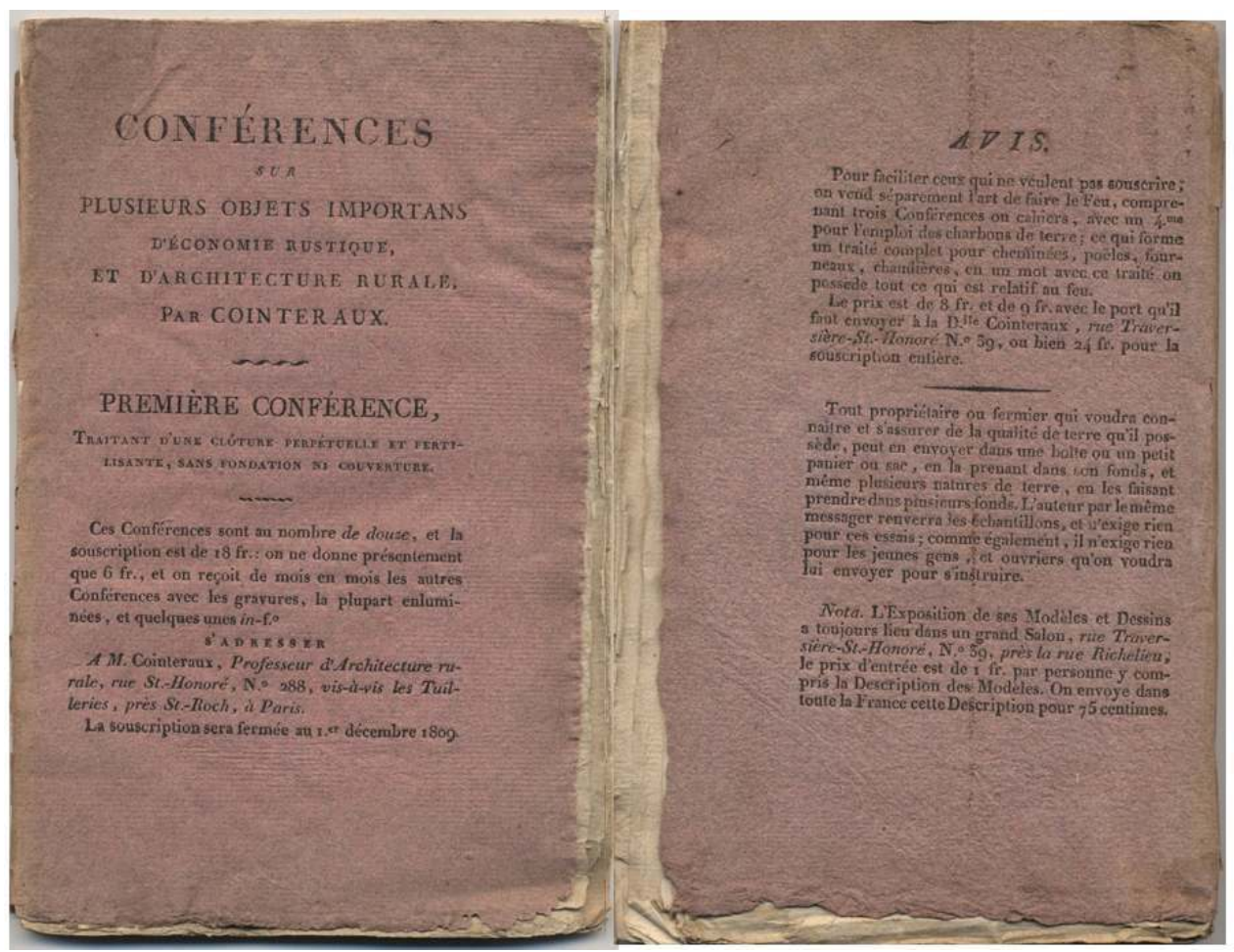

Avis commerciaux sur les couvertures imprimées des Conférences de 1809 (cat. 54)

REPRO. GARRIC, JEAN-PHILIPPE. @ JEAN-PHILIPPE GARRIC.

Les modalités de vente qu'il propose sont parfois plus singulières, comme celle qui consiste à offrir l'opuscule sur l'Odéon aux acheteurs de l'ouvrage sur Paris ou à regrouper dix-huit publications antérieures formant peu ou prou ses œuvres complètes en deux volumes proposés à moitié prix. Cette proposition qui intervient en 1809 témoigne du fait que la plupart de ses anciens titres étaient encore disponible. Elle ne semble pas avoir rencontré un succès considérable car nous n'avons identifié qu'un seul exemplaire de ce recueil de textes (cat. 50). Mais son inventivité commerciale ne s'arrêtait pas là. Ainsi propose-t-il dans son ouvrage sur les nouveaux poids et mesures judicieusement intitulé "L'Arithmétique républicaine», une offre qui l'est tout autant puisqu'elle propose de substituer les onze à la dizaine aux anciens treize à la douzaine (cat.13). Dans un autre ouvrage on découvre avec un étonnement sceptique que Cointeraux aurait eu des concessionnaires (cat. 44). L'annonce de la parution de son traité d'agritecture, dont il précise entre parenthèses qu'il s'agit d'une "nouvelle science", conjugue plusieurs données intéressantes. D'une part la confusion entre un journal, un périodique, et un traité, forme finie et stable par excellence, qui s'accompagne de la notion de souscripteur. Cointeraux cherchait en permanence à fidéliser une clientèle en créant des séries, des abonnements. Ensuite l'annonce de cet ouvrage est placée sous le signe de « Nouvelles conditions et rabais », qui comprend un ultimatum (fig. $\mathbf{n}^{\circ}$ ). 


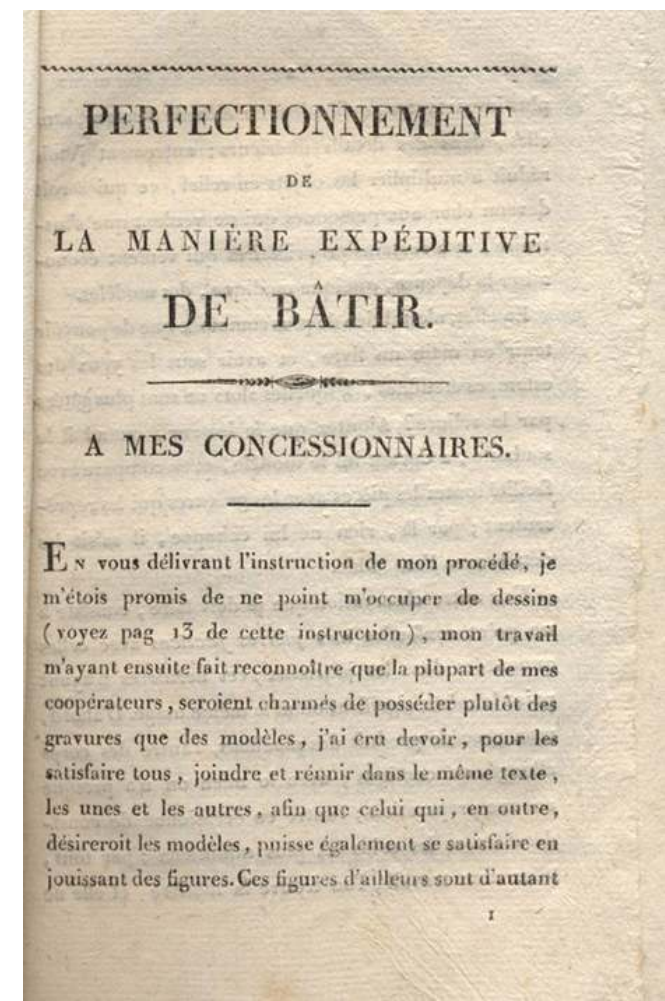

Perfectionnement de la manière expéditive de bâtir. À mes concessionnaires (cat. 44)

REPRO. GARRIC, JEAN-PHILIPPE. @ JEAN-PHILIPPE GARRIC.

\section{La fabrication des ouvrages}

En revanche, elle renvoie aux modalités très artisanales de fabrication de ses propres ouvrages. S'il en assurait lui-même la commercialisation directe ou par correspondance, il faisait appel à des imprimeurs dont les signatures jointes à l'observation des papiers et des fontes typographiques utilisés, permettent de constater différentes manipulations. Ainsi le Traité de la construction des manufactures et des maisons de campagne de 1791 (cat. 2.C), qui forme la troisième partie, de l'École d'architecture rurale (cat. 2) comprend-il une page de titre avec la mention "chez l'auteur et chez Niodot», une première partie avec la mention «de l'imprimerie nationale» et une seconde partie avec l'indication «De l'imprimerie de Vézard \& Le Normant ", la pagination elle-même irrégulière et l'usage de différents papiers démontre que ces volumes ont été fabriqués à partir de cahiers initialement imprimés séparément et réassemblés un à un manuellement. Il existe également des exemplaires de la quatrième partie de la même École d'architecture rurale (cat. 2.D) datés 1806 dont le titre porte au recto une note de l'éditeur qui précise : «Ce cahier dépendant de la collection du Pisé, publié en 1791, et réimprimé en 1803, et en 1806... ». Mais la page de titre et le dernier feuillet sont imprimés sur des papiers différents et avec une fonte qui n'est pas la même. Il s'agit en fait d'exemplaires de remise en vente fabriqués à partir d'exemplaires du premier tirage qui portait à l'origine la date de novembre 1791, avec une fausse mention de $3^{\mathrm{e}}$ édition destinée à accréditer le succès commercial de l'ouvrage. La même chose est vraie pour l'exemplaire consulté de L'Art de 
peindre à fresque sur le pisé (cat. 17), qui donne Paris comme lieu d'édition et porte la mention «Publié de rechef en l'an 11 ou 1803 », tandis que l'intertitre placé au début de la seconde partie indique Lyon comme lieu d'édition. Les deux parties étant pourtant imprimées sur un même papier à l'exclusion du titre de la première partie, il s'agit très probablement une nouvelle fois d'un titre de remise en vente. L'ouvrage sur Paris de 1799 porte pour sa part deux différents noms d'imprimeurs pour ses deux différentes parties: un indice supplémentaire du caractère extrêmement artisanal de cette fabrication des livres, auquel s'ajoute la présence de planches «enluminées » manuellement, dont la confection devait, on l'imagine mobiliser toute la famille Cointeraux à la veillée.

\section{Le choix des titres : une littérature événement}

Le néologisme le plus brillant inventé par Cointeraux est certainement " Agritecture ». Il correspond cependant à une conviction partagée à l'époque, Toussaint, par exemple, commençant en 1810 son traité de géométrie et d'architecture par cette forte déclaration: "L'Architecture est après l'agriculture le premier et le plus utile des arts ». Apparemment il s'en délecte et projette effectivement de fondre les deux disciplines en un unique enseignement. En 1806, il intitule l'un de ses opuscules sur le chauffage Avis au peuple sur l'économie de son bois (cat. 33). Il s'agit d'une référence - qui ne pouvait alors passer inaperçue - au célébrissime ouvrage de Samuel Auguste Tissot, Avis au peuple sur sa santé, publié en 1761, qui avait depuis lors été traduit en dix-sept langues. Le médecin suisse y dénonçait les dangers de la masturbation. D’autres références, faites à des livres moins connus, témoignent d'une certaine culture livresque, sans qu'on soit en mesure de dire si ce genre d'imitation avait ou non une finalité ironique, comme L'Art de diminuer et de perdre son bien et sa tranquillité, ou les Propriétaires au confessionnal (cat.33), manifestement inspiré de l'anonyme L'Art d'augmenter et de conserver son bien, ou règles générales pour l'administration d'une terre, publié à Amsterdam en 1784. Faut-il voir, par exemple, une forme de provocation consciente dans le fait d'intituler un fascicule de cinquante six pages Supplément au cours complet d'agriculture, de l'abbé Rozier (cat. 51), qui rappelons-le compte dix épais volumes in $-4^{\circ}$, ou une naïveté commerciale dans le fait d'ajouter «Ouvrage indispensable aux pocesseurs de toutes les anciennes éditions, in- $4^{\circ}$. Aux possesseurs des nouvelles éditions, in- $8^{\circ}$. Et utiles à tous ceux qui ne possèdent aucune de ces éditions »?

Il est également vraisemblable que la conférence de 1809 qui traite notamment de De la théorie de la surface du globe terrestre appliquée aux besoins de l'homme (cat.54.C) et qui s'inspire, sans le citer, des travaux de Lavoisier, soit plus directement redevable à l'ouvrage que Charles Gilbert Morel de Vindé avait signé en 1797 sous le titre Des Révolutions du Globe. Conjecture formée d'après les Découvertes de Lavoisier sur la décomposition et la recomposition de l'eau et qu'il avait réédité en 1806.

\section{Une littérature événement}

Une autre originalité des publications de Cointeraux est leur lien permanent avec des événements ou des manifestations publiques: expositions, conférences, écoles, ce qui donne des titres comme "École d'architecture rurale», ou "Conférences». Plus globalement ses ouvrages peuvent être analysés comme une mise en scène de lui-même. 
En effet, pour modestes et pratiques que puissent être ses sujets de prédilection, il n'en développe pas moins dans ses publications une dimension autobiographique, qui tient à la fois au rôle de démonstrateur et de cobaye de ses propres idées qu'il joue en permanence, à la fonction d'autopromotion sociale qu'il assigne à ses publications et à la confusion qu'il entretient entre les différentes facettes de son activité qui surprime toute distance entre sa vie privée et sa vie publique. Ainsi informe-t-il aussi bien le public du fait qu'il fait peser sur sa famille le poids de son activité, que des événements qui le touchent, parmi lesquels le décès de son épouse: les lecteurs de Cointeraux sont un peu de sa famille.

\section{Le style Cointeraux}

Mais Cointeraux est également porté à utiliser des formes littéraires plutôt inattendues dans le cadre d'un ouvrage technique, comme à forger de brillants néologismes. Ainsi utilise-t-il, dans l'ouvrage qu'il consacre en 1797 à la manière de peindre à fresque sur le pisé, la forme d'un dialogue entre les deux principaux matériaux concernés : la peinture et le pisé (cat. 17). Les deux techniques ainsi personnalisées dissertent de leurs mérites relatifs, permettant à l'auteur de parler de lui-même par leur voix à la troisième personne.

Extrait :

«Le Pisé : ô ma divine maîtresse! Je n'aurais jamais pu croire qu'il nous eut été aussi facile d'orner la demeure des humains.

«La peinture : Ne crains point d'ajouter qu'il nous est également aisé de varier leurs plaisirs, et de leur en procurer une infinité de nouveaux? Non seulement les hommes auront avec nous la faculté de faire dans leurs jardins toutes sortes de sujets plus agréables les uns que les autres, tels que des piédestaux, des obélisques, des colonnes triomphales; mais plus encore ils pourront faire avec le pisé des figures humaines.

«Le pisé : Cela paraît impossible à l'égard des figures.

«La peinture: Je n'entreprendrai pas à présent de t'en donner la certitude; j'attends pour t'en convaincre, que le professeur aie fait dans son école une de ces figures comme il en a le projet ».

Cointeraux, décidément très en verve, invente dans la suite du même texte ni plus ni moins qu'une nouvelle science (le débat tourne toujours autour de la question de l'ornement des jardins) :

«La peinture : Comment, tu aurais encore la témérité de vouloir faire de plus beaux jardins que ceux connus sous le titre pompeux de jardins anglais?

«Le pisé : N'en doute point. Sans copier, comme l'ont fait certains milords, toutes les productions chinoises, je me flatte d'être original, et de produire des vérités ainsi que des utilités. Un auteur a dit : le beau sera toujours beau; il n'est donc pas question de rejeter entièrement la ligne droite, comme l'ont fait les prétendus et fameux jardiniers anglais; d'ailleurs, il est ici plus question d'adopter une nouvelle science qui puisse enseigner la vraie route à suivre.

«La peinture : Tu m'étonnes! Quelle est cette nouvelle science?

«Le pisé : L'AGRITECTURE.»

La suite de l'ouvrage est consacrée à défendre cette science nouvelle et se termine par une « Invocation au directoire exécutif » qui commence en ces termes :

"L'Architecture, de tous les temps, a été traitée isolément: l'Agriculture, de tous les temps, a été expliquée séparément. C'est une erreur, ces deux arts ne sauraient être approfondis, qu'en fondant leurs principes dans le même creuset de l'esprit ; il en résultera une science nouvelle que je nomme avec fondement Agritecture.» 
Le texte se termine par un appel éploré et emphatique à l'aide :

« Ô VOUS MEMBRES ÉCLAIRÉS DU DIRECTOIRE! daignez souscrire au journal d'une nouvelle science »; "Et vous, MINISTRE DE LA GUERRE, choisi par une voix unanime, laisserez-vous dans un éternel oubli des moyens et des procédés aussi avantageux ».

29 Au fil des années et des fascicules successifs, l'auteur s'affirme, révélant une véritable maîtrise et un plaisir du verbe :

« Les vignes sont belles, et ma vie passe, et les vendanges approchent (1806, juin). Il est tems que je publie un de ces procédés rares et avantageux dont je suis sur pour l'avoir pratiqué ", ajoute-t-il. Écrit-il dans De la nécessité de dégrapper les raisins (cat. 40).

Témoignant même d'une réflexion sur le style le mieux approprié pour soutenir son engagement :

« je me servirai du langage le plus simple, pour mettre mes leçons à la portée de tout le monde, surtout des cultivateurs qui y sont les plus intéressés. Quand il s'agit de la pratique, ou de la main d'œuvre, on ne doit point embrouiller la pensée par des mots scientifiques, propres à jeter le lecteur dans l'embarras ; car la confusion des idées est un grand obstacle aux connaissances que l'on cherche à acquérir, et empêche souvent les procédés et les méthodes les plus utiles, et l'exécution la plus aisée de réussir. Qu'importent les belles phrases aux propriétaires... »

Ce qui ne lui interdit pas pourtant de se faire lyrique et cosmogonique :

«Vénus, dite l'étoile du berger, est admirable par son éclat et par la grande lumière qu'elle nous envoie, aussi a-t-elle été remarquée de tout temps par les gens de la campagne.

Le soleil, astre du jour, est le principal agent de l'art agricole: avec lui tout prospère, sans lui tout dépérit.

Voilà les seuls objets de la sphère qui nous intéressent. À quoi sert à de faibles humains la connaissance d'une machine aussi compliquée, et composée de cent et cent millions de mondes?»

Ou encore :

« Le printemps s'annonce, et voilà la nature prête à s'habiller. D'abord tous les êtres vivants, soit hommes soit animaux, éprouvent la joie la plus vive, et toutes les plantes ressentant les effets d'une végétation universelle commencent à se développer. Le mouvement continu des cieux auquel notre soleil participe, fait que toutes les surfaces plates, obliques, proéminentes, en un mot irrégulières de ce globe, se trouvent successivement pénétrées d'une chaleur bienfaisante » (cat. 54). bâtir aux champs, ou mieux encore, l'Agritecture considérée comme un des beaux-arts.

\section{NOTES}

1. - Voir infra. Le néoclassicisme n'est pas un classicisme. Une mutation dans les livres d'architecture, p. 204.

2. - De QUINCEY, Thomas. "Jeanne d'Arc ». Dans De l'Assassinat considéré comme un des Beaux-Arts. Paris : Gallimard, 1963, p. 244 (1 ère éd. anglaise, 1847, dans le Tait's Magazine). 
3. - SIMOND, Louis. Voyage en Angleterre, pendant les années 1810 et $1811 . .$. Paris : Treuttel et Wurtz, 1817, p. 379.

4. - Dans sa conférence introductive au colloque L'art de bâtir aux champs, le 14 octobre 2010 à l'Institut national d'histoire de l'art.

5. - COINTERAUX, François. De la distribution des batimens de pisé, p. 4.

6. - BARIDON, Laurent. «Le pisé de François Cointeraux (1740-1830) : la terre comme utopie ». Dans CARVAIS, Robert, GUILLERME, André, NEGRE, Valérie et SAKAROVITCH, Joël (dir.). Édifice et artifice. Histoires constructives. Paris : Picard, 2010, p. 101-108.

7. - CELLAURO, Louis et RICHAUD, Gilbert. « Thomas Jefferson and François Cointeraux, Professor of Rural Architecture in Revolutionnary Paris ». Architectural History, 48, 2005, p. 173-206 ; «François Cointeraux's école d'architecture rurale (1790-1791) and its influence in Europe and in the Colonies ». Architectural History, 49, 2006, p. 129-148.

\section{RÉSUMÉS}

François Cointeraux (1740-1830), selon ses propres termes, avait fait « remuer à la fois la truelle \& le marteau, avec la bêche et la houe ». Il est ainsi l'un des rares théoriciens de l'architecture rurale qui puisse se vanter d'appartenir à la classe laborieuse et n'aborde donc pas la question du point de vue surplombant du grand propriétaire, de l'agronome ou de l'architecte, mais d'un point de vue proche du terrain. Pourtant, s'il peut revendiquer se statut qui le distingue et le rapproche de son objet, son activité ne se résume pas à ses visées pratiques. Elle dispute aux élites leur autorité intellectuelle et culturelle sur le monde rural et mobilise pour cela un vrai talent littéraire, un sens certain de la formule, voire de la publicité. Au début d'un siècle où les publications spécialisées en architecture rurale se multiplient, Cointeraux est l'un des premiers à investir ce domaine et, bien que son sujet de prédilection ait été la construction en terre crue, ses centres d'intérêt multiples s'étendaient à toutes les questions touchant à la vie et au travail des agriculteurs.

According to his own expression, François Cointeraux (1740-1830) had wielded « both trowel and hammer, and both spade and hoe ». He is consequently one of the rare theoreticians of rural architecture who can claim to belong to the labouring classes and who can approach the question not from the superior viewpoint of the great landowner, the agronomist or the architect, but from a point of view close to the terrain. Nonetheless, if he can make a claim to this status which distinguishes him from other observers and which puts him in closer proximity with his subject, his activity is not limited to merely practical considerations. He is in conflict with the elites as to their intellectual and cultural authority in the rural world, and he brings to his arguments a real literary talent, a certain sense of the formula and even of publicity. At the beginning of a century when publications specialising in rural architecture were beginning to become more frequent, Cointeraux is one of the first to occupy the field, and although his main centre of interest is in constructions made out of rammed earth; his other interests are many and varied and address all the questions concerning the life and work of the farmer. 


\section{AUTEUR}

\section{JEAN-PHILIPPE GARRIC}

Conseiller scientifique à l'INHA pour l'histoire de l'architecture jean-philippe.garric@inha.fr 